\title{
On the binary codes with parameters of doubly-shortened 1-perfect codes
}

\author{
Denis S. Krotov
}

October 24, 2018

\begin{abstract}
We show that any binary $\left(n=2^{m}-3,2^{n-m}, 3\right)$ code $C_{1}$ is a part of an equitable partition (perfect coloring) $\left\{C_{1}, C_{2}, C_{3}, C_{4}\right\}$ of the $n$-cube with the parameters $((0,1, n-1,0)(1,0, n-1,0)(1,1, n-4,2)(0,0, n-1,1))$. Now the possibility to lengthen the code $C_{1}$ to a 1 -perfect code of length $n+2$ is equivalent to the possibility to split the part $C_{4}$ into two distance-3 codes or, equivalently, to the biparticity of the graph of distances 1 and 2 of $C_{4}$. In any case, $C_{1}$ is uniquely embeddable in a twofold 1-perfect code of length $n+2$ with some structural restrictions, where by a twofold 1 -perfect code we mean that any vertex of the space is within radius 1 from exactly two codewords.
\end{abstract}

The hypercube $H^{n}=\left(V\left(H^{n}\right), E\left(H^{n}\right)\right)$ of dimension $n$ is the graph whose vertices are the all binary $n$-words, two words being adjacent if and only if they differ in exactly one position.

$d(\cdot, \cdot)$ - the Hamming distance, i.e., the natural graph distance in $H^{n}$.

$\overline{0}=0 \ldots 0$ (the all-zero word), $\overline{1}=1 \ldots 1$ (the all-one word).

A binary code $C$ of length $n$ and code (or minimal) distance $d$, or $(n,|C|, d)$ code, is a subset of $V\left(H^{n}\right)$ such that $d(\bar{x}, \bar{y}) \geq d$ for any different $\bar{x}$ and $\bar{y}$ from $C$.

A partition $\left\{C_{1}, \ldots, C_{r}\right\}$ of $V\left(H^{n}\right)$ into $r$ nonempty parts is said to be equitable with parameters $\left(S_{i j}\right)_{i, j=1}^{n}$ if for every $i, j \in\{1, \ldots, r\}$ every vertex $\bar{x}$ from $C_{i}$ has exactly $S_{i j}$ neighbors from $C_{j}$ (the corresponding $r$-valued function on $V\left(H^{n}\right)$ is known as a perfect coloring).

A binary code $C \subset V\left(H^{n}\right)$ is said to be 1-perfect if every vertex $\bar{x} \in V\left(H^{n}\right)$ is at the distance 0 or 1 from exactly one codeword. Equivalently, $\left\{C, V\left(H^{n}\right) \backslash C\right\}$ is an equitable partition with parameters $((0, n)(1, n-1))$. Equivalently, $C$ is a $\left(2^{m}-1,2^{2^{m}-m-1}, 3\right)$ code, $n=2^{m}-1$.

We will say that a multiset $B \subset V\left(H^{n}\right)$ is a twofold 1-perfect code if every vertex $\bar{x} \in V\left(H^{n}\right)$ is at the distance 0 or 1 from exactly two codewords of $B$. We will say that a multiset $B \subset V\left(H^{n}\right)$ is splittable if it can be represented as the (multiset) union of two distance- 3 codes; otherwise $B$ is unsplittable. The existence of unsplittable twofold 1-perfect codes was proved in [6].

We say that a code $C^{\prime}$ if obtained by shortening from a code $C \subset V\left(H^{n}\right)$ if $C^{\prime}=\left\{\bar{x} \in V\left(H^{n-1}\right) \mid \bar{x} 0 \in C\right\}$. Respectively, $C^{\prime \prime}$ is doubly-shortened from $C$ if 
$C^{\prime \prime}=\left\{\bar{x} \in V\left(H^{n-2}\right) \mid \bar{x} 00 \in C\right\}$. (Here and elsewhere, for $\bar{x}=x_{1} x_{2} \ldots x_{n}$, by $\bar{x} 0$ we mean the concatenation of $\bar{x}$ with 0 , i.e., the word $x_{1} x_{2} \ldots x_{n} 0$; similarly we define $\bar{x} 1, \bar{x} 00, \bar{x} 01, \ldots$; we also expand this notation for sets of words, e.g., $C 0=\{\bar{x} 0 \mid \bar{x} \in C\}$.)

It is known [1] that shortened and doubly-shortened (and even triply-shortened) 1-perfect codes have the maximal cardinality among all the codes of the same length and code distance 3 . The question [4] is: can every code with such parameters $\left(\left(2^{m}-2,2^{2^{m}-m-2}, 3\right)\right.$ or $\left.\left(2^{m}-3,2^{2^{m}-m-3}, 3\right)\right)$ be represented as a shortened or doubly-shortened 1-perfect code?

For $\left(2^{m}-2,2^{2^{m}-m-2}, 3\right)$ codes the question is solved [2]. In fact, such a code $C_{1}$ generates an equitable partition $\left\{C_{1}, C_{2}, C_{3}\right\}$ with parameters $((0, n, 0)(1, n-$ $2,1)(0, n, 0))$. Then, the code

$$
C=C_{1} 0 \cup C_{3} 1
$$

is 1 -perfect.

In this paper we prove that a $\left(2^{m}-3,2^{2^{m}-m-3}, 3\right)$ code $C_{1}$ generates an equitable partition $\left\{C_{1}, C_{2}, C_{3}, C_{4}\right\}$ with parameters $((0,1, n-1,0)(1,0, n-$ $1,0)(1,1, n-4,2)(0,0, n-1,1))$. If the code $C_{4}$ is splittable into two distance- 3 codes $C^{\prime}$ and $C^{\prime \prime}$, then the code

$$
C=C_{1} 00 \cup C_{2} 11 \cup C^{\prime} 01 \cup C^{\prime \prime} 10
$$

is 1-perfect. However, the problem of splittability of $C_{4}$ remains open. So, the problem of embedding $C_{1} 00$ in a 1-perfect code is unsolved; although, $C_{1} 00$ is proved to be embedded in twofold 1-perfect codes

$$
2 \times C_{1} 00 \cup 2 \times C_{2} 11 \cup C_{4} 01 \cup C_{4} 10
$$

and

$$
C_{1} 00 \cup C_{2} 00 \cup C_{1} 11 \cup C_{2} 11 \cup C_{4} 01 \cup C_{4} 10
$$

(Theorems 2 and [3), whose splittability is equivalent to the splittability of $C_{4}$.

\section{Notation and basic facts}

Let $C_{1}$ be a binary code of length $n=2^{m}-3$, cardinality $2^{n-m}$, and minimal distance 3 .

Denote

$$
\begin{gathered}
C_{2}=C_{1}+\overline{1}=\left\{\bar{x} \mid \bar{x}+\overline{1} \in C_{1}\right\}, \\
C_{3}=\left\{\bar{x} \mid d\left(\bar{x}, C_{1}\right)=1\right\} \backslash C_{2}, \\
C_{4}=V\left(H^{n}\right) \backslash\left(C_{1} \cup C_{2} \cup C_{3}\right) ; \\
A_{l}^{j}(\bar{x})=\left|\left\{\bar{y} \in C_{j} \mid d(\bar{x}, \bar{y})=l\right\}\right|, \quad j \in\{1,2,3,4\}, \bar{x} \in V\left(H^{n}\right)
\end{gathered}
$$

(the tuple $\left(A_{0}^{i}(\bar{x}), A_{1}^{i}(\bar{x}), \ldots, A_{n}^{i}(\bar{x})\right)$ is known as the weight distribution of $C_{i}$ with respect to $\bar{x})$, 


$$
\bar{A}_{l}^{i j}=\frac{1}{\left|C_{i}\right|} \sum_{\bar{x} \in C_{i}} A_{l}^{j}(\bar{x}), \quad i, j \in\{1,2,3,4\}, l \in\{0, \ldots, n\}
$$

(the tuple $\left(\bar{A}_{0}^{i i}, \bar{A}_{1}^{i i}, \ldots, \bar{A}_{n}^{i i}\right)$ is known as the inner distance distribution of $\left.C_{i}\right)$.

Best and Brouwer [1] showed that $\left(2^{m}-3,2^{n-m}, 3\right)$ codes are optimal, i.e. any $\left(2^{m}-3, M, 3\right)$ code satisfies $M \leq 2^{n-m}$. Moreover,

Lemma 1 [1]. The inner distance distribution $\left(\bar{A}_{l}^{11}\right)_{l=0}^{n}$ does not depend on the choice of the $\left(2^{m}-3,2^{n-m}, 3\right)$ code $C_{1}$.

We will also need the following fact:

Lemma 2. Any 1-perfect or twofold 1-perfect code $C$ is antipodal; i.e., in multiset terms, for any $\bar{x} \in V\left(H^{n}\right)$ the $C$-multiplicities of $\bar{x}$ and $\bar{x}+\overline{1}$ coincide.

In the case of 1-perfect codes this is well-known fact, which follows from the results [8, 12. For twofold 1-perfect codes, the fact has a similar proof. Alternatively, Lemma 2 follows from the fact that the multiplicity function of the considered code is, up to an additive constant, an eigenfunction of $H^{n}$ with the eigenvalue -1 and the corresponding eigenspace has a simple basis from antipodal functions.

\section{An element of equitable partition}

Proposition 1. If $C_{1}$ is a doubly-shortened 1-perfect code of length $n$, then $\bar{A}_{n}^{11}=\bar{A}_{1}^{24}=\bar{A}_{1}^{42}=0, \bar{A}_{n-1}^{11}=\bar{A}_{1}^{44}=1$, and $A_{1}^{4}(\bar{x})=\bar{A}_{1}^{34}=2$ for any $\bar{x} \in C_{3}$.

Proof: Let $C=C_{1} \times\{00\} \cup C^{\prime} \times\{01\} \cup C^{\prime \prime} \times\{01\} \cup C^{\prime \prime \prime} \times\{11\}$ be a 1-perfect code. If $\bar{x} \in C_{1}$ (i.e. $\bar{x} 00 \in C$ ), then $\bar{x} 00+\overline{1} \in C$, i.e., $\bar{x}+\overline{1} \in C^{\prime \prime \prime}$; so, $C_{2}=C^{\prime \prime \prime}$ and $\bar{A}_{n}^{11}=0$.

If a vertex $\bar{y}$ is at distance at least 2 from $C_{1}$, then, by the definition of a 1-perfect code, the vertex $\bar{y} 00$ is at distance 1 from an element of $C$, which is either $\bar{y} 01$ or $\bar{y} 10$. So, $\bar{y} \in C^{\prime} \cup C^{\prime \prime}$. Vise versa, any $\bar{y} \in C^{\prime} \cup C^{\prime \prime}$ is at distance at least 2 from $C_{1}$, because the minimal distance of $C$ is 3 . So, $C_{4}=C^{\prime} \cup C^{\prime \prime}$.

Because of the minimal distance of $C$, the sets $C_{2}=C^{\prime \prime \prime}$ and $C_{4}=C^{\prime} \cup C^{\prime \prime}$ are at distance more than 1 from each other. This means $\bar{A}_{1}^{24}=\bar{A}_{1}^{42}=0$.

We state that for any $\bar{x}$ from $C_{1}$ there is exactly one vertex of $C_{2}$ at the distance 1 from $\bar{x}$. Indeed, the vertex $\bar{x} 11$ from $V\left(H^{n+2}\right)$ is at the distance 1 from exactly one codeword of $C$, which can be only of type $\bar{y} 11$, where $\bar{y} \in C_{2}$ and $d(\bar{x}, \bar{y})=1$. Then the vertex $\bar{y}+\overline{1}$ is the only $C_{1}$-vertex at the distance $n-1$ from $\bar{x}$; so, $\bar{A}_{n-1}^{11}=1$. The remaining part of the proposition is proved by similar arguments. $\triangle$

We will first prove that

Lemma 3. All the numbers $\bar{A}_{l}^{i j}(i, j \in\{1,2,3,4\}, l \in\{0, \ldots, n\})$ do not depend on the choice of the $\left(2^{m}-3,2^{n-m}, 3\right)$ code $C_{1}$. 
Proof: Once we have proved that $\bar{A}_{l}^{i j}$ does not depend on the choice $C_{1}$, we know that it is the same as if $C_{1}$ would be a double-shortened 1-perfect (for example, Hamming) code. Moreover if it is equal to the minimal or maximal possible value of $A_{l}^{j}(\bar{x}), \bar{x} \in C_{i}$, then $A_{l}^{j}(\bar{x})=\bar{A}_{l}^{i j}$ for any $\bar{x} \in C_{i}$.

In particular, for any $\bar{x} \in C_{1}$

$$
A_{n}^{1}(\bar{x})=0 \quad \text { and } \quad A_{n-1}^{1}(\bar{x})=1 .
$$

This means that the sets $C_{1}$ and $C_{2}$ are disjoint and

any vertex from $C_{2}$ has exactly one neighbor from $C_{1}$, and vise versa

(the fact (44) will be used later). So, $\left\{C_{1}, C_{2}, C_{3}, C_{4}\right\}$ is a partition of $V\left(H^{n}\right)$, and we can derive relations between the cardinalities of $C_{i}$ :

$\left|C_{2}\right|=\left|C_{1}\right|, \quad\left|C_{3}\right|=(n-1)\left|C_{1}\right|, \quad\left|C_{4}\right|=\left|V\left(H^{n}\right)\right|-\left|C_{1}\right|-\left|C_{2}\right|-\left|C_{3}\right|=2\left|C_{1}\right|$.

Now we claim the following:

$$
\begin{aligned}
\bar{A}_{l}^{i 2} & =\bar{A}_{n-l}^{i 1} \\
\bar{A}_{l}^{i 3} & =(n-l+1) \cdot \bar{A}_{l-l}^{i 1}+(l+1) \cdot \bar{A}_{l+l}^{i 1}-\bar{A}_{l}^{i 3} \\
\bar{A}_{l}^{i 4} & =\left(\begin{array}{c}
n \\
l
\end{array}\right)-\bar{A}_{l}^{i 1}-\bar{A}_{l}^{i 2}-\bar{A}_{l}^{i 3} \\
\left|C_{i}\right| \cdot \bar{A}_{l}^{i j} & =\left|C_{j}\right| \cdot \bar{A}_{l}^{j i}
\end{aligned}
$$

Indeed, (5) follows from $A_{l}^{2}(\bar{x})=A_{n-l}^{1}(\bar{x})$, which is straightforward from the definition of $C_{2}$; (6) follows from $A_{l}^{3}(\bar{x})=(n-l+1) \cdot A_{l-l}^{1}(\bar{x})+(l+1) \cdot A_{l+l}^{1}(\bar{x})-$ $A_{l}^{3}(\bar{x})$, which is straightforward from the definition of $C_{3}$ and (4); (7) follows from

$$
A_{l}^{4}(\bar{x})+A_{l}^{1}(\bar{x})+A_{l}^{2}(\bar{x})+A_{l}^{3}(\bar{x})=\left(\begin{array}{c}
n \\
l
\end{array}\right),
$$

which is from the fact that $\left\{C_{1}, C_{1}, C_{1}, C_{1}\right\}$ is a partition of $V\left(H^{n}\right)$; the right and left part of (7) are just different ways to calculate the cardinality of $\{(\bar{x}, \bar{y}) \mid \bar{x} \in$ $\left.C_{i}, \bar{y} \in C_{j}, d(\bar{x}, \bar{y})=l\right\}$.

Starting from $\bar{A}_{l}^{11}$, we can calculate $\bar{A}_{l}^{1 j}$ by (5) (7), the values of $\bar{A}_{l}^{j 1}$ by (8), the values of $\bar{A}_{l}^{j i}$ by (5-7); so, Lemma 3 is proved. $\triangle$

Theorem 1. The partition $\left\{C_{1}, C_{2}, C_{3}, C_{4}\right\}$ of $V\left(H^{n}\right)$ is equitable with parameters

$$
\left(S_{i j}\right)_{i, j=1}^{4}=\left(\begin{array}{cccc}
0 & 1 & n-1 & 0 \\
1 & 0 & n-1 & 0 \\
1 & 1 & n-4 & 2 \\
0 & 0 & n-1 & 1
\end{array}\right) .
$$

Proof: Assume $i, j \in\{1,2,3,4\}, \bar{x} \in C_{i}$. We will show that $A_{1}^{j}(\bar{x})=S_{i j}$. 
We have already found (4) that $A_{1}^{j}(\bar{x})=1$ if $(i, j) \in\{(1,2),(2,1)\}$. Since $C_{1}$ and $C_{2}$ are distance- 3 codes, $A_{1}^{j}(\bar{x})=0$ if $(i, j) \in\{(1,1),(2,2)\}$. Then, by the definition of $C_{4}$, we have $A_{1}^{j}(\bar{x})=0$ if $(i, j) \in\{(1,4),(4,1)\}$.

By (9) we get $A_{1}^{j}(\bar{x})=n-0-1-0=n-1$ if $(i, j)=(1,3)$.

Since $\bar{A}_{1}^{24}=\bar{A}_{1}^{42}=0$, we also have $A_{1}^{j}(\bar{x})=0$ if $(i, j) \in\{(2,4),(4,2)\}$.

By (9), $A_{1}^{j}(\bar{x})=n-1-0-0=n-1$ if $(i, j)=(2,3)$.

Let us check that $A_{1}^{j}(\bar{x})=1$ if $(i, j)=(4,4)$. Since $\bar{A}_{1}^{44}=1$ (Proposition 1), it is enough to prove that $A_{1}^{j}(\bar{x})$ is odd. Indeed, as follows from the arguments above, the neighborhood of $\bar{x}$ consists of only $C_{3}$ - and $C_{4}$-vertices. Every such $C_{3}$-vertex is adjacent with exactly one $C_{1}$-vertex, which is at distance 2 from $\bar{x}$. While every such $C_{1}$-vertex is adjacent with exactly two vertices from the neighborhood of $\bar{x}$. So, this neighborhood contains an even number of vertices from $C_{3}$ and, consequently, an odd, from $C_{4}$.

Automatically, we get $A_{1}^{j}(\bar{x})=n-0-0-1=n-1$ if $(i, j)=(4,3)$.

Let us show that $A_{1}^{j}(\bar{x})=2$ if $(i, j)=(3,4)$. We will calculate the number $T$ of triples $\{\bar{a}, \bar{b}, \bar{c}\}$ such that $\bar{b} \in C_{3}$ is adjacent to both $\bar{a}, \bar{c} \in C_{4}$. At first, we observe that $T=\left|C_{4}\right| \bar{A}_{2}^{44}$ is independent on the choice of $C_{1}$. At second, it can be calculated as

$$
\sum_{\bar{b} \in C_{3}} \frac{A_{1}^{4}(\bar{b})\left(A_{1}^{4}(\bar{b})-1\right)}{2}=\frac{1}{2} \sum_{\bar{b} \in C_{3}}\left(A_{1}^{4}(\bar{b})\right)^{2}-\frac{1}{2}\left|C_{3}\right| \bar{A}_{1}^{34}
$$

so, by the Cauchy-Bunyakovsky inequality,

$$
T \geq \frac{1}{2\left|C_{3}\right|}\left(\sum_{\bar{b} \in C_{3}} A_{1}^{4}(\bar{b})\right)^{2}-\frac{\left|C_{3}\right|}{2} \bar{A}_{1}^{34}=\frac{\left|C_{3}\right|}{2} \bar{A}_{1}^{34}\left(\bar{A}_{1}^{34}-1\right),
$$

where the equality holds if and only if all $A_{1}^{4}(\bar{b}), \bar{b} \in C_{3}$, are equal to the same value (i.e., to $\bar{A}_{1}^{34}=2$ ). But the last is true when $C_{1}$ is a doubly-shortened 1-perfect code (Proposition 1); consequently, it is true for any $\left(2^{m}-3,2^{n-m}, 3\right)$ code.

Finally, if $(i, j)=(3,3)$, then $A_{1}^{j}(\bar{x})=n-1-1-2=n-4 . \triangle$

Remark 1. 1) If we unify the two parts $C_{1}$ and $C_{2}$, say $C_{12}=C_{1} \cup C_{2}$, then we will obtain an equitable partition $\left\{C_{12}, C_{3}, C_{4}\right\}$ with parameters

$$
\left(\begin{array}{lll}
1 & n-1 & 0 \\
2 & n-4 & 2 \\
0 & n-1 & 1
\end{array}\right) .
$$

We see that the parameter matrix is symmetrical with respect to interchanging of the parts $C_{12}$ and $C_{4}$. But $C_{12}$ is known to be splittable, while the splittability of $C_{4}$ is questionable. When $C_{1}$ is a doubly-shortened 1-perfect code, we know that both $C_{12}$ and $C_{4}$ are splittable. Moreover, one can construct an equitable partition with parameters (10) whose first and third parts are unsplittable. The problem is if there exists such a partition with exactly one of $C_{12}$ and $C_{4}$ being splittable. 
2) If $C_{4}$ is splittable, then after splitting it, from the partition $\left\{C_{1}, C_{2}, C_{3}, C_{4}\right\}$ we obtain an equitable partition with parameters

$$
\left(\begin{array}{lllll}
0 & 1 & n-1 & 0 & 0 \\
1 & 0 & n-1 & 0 & 0 \\
1 & 1 & n-4 & 1 & 1 \\
0 & 0 & n-1 & 0 & 1 \\
0 & 0 & n-1 & 1 & 0
\end{array}\right)
$$

which also have some obvious symmetries.

Remark 2. An equitable partition with $\left\{C_{12}, C_{3}, C_{4}\right\}$ of $H^{n}$ with parameters (10) generates an equitable partition $\left\{G_{1}, G_{2}, G_{3}, G_{4}\right\}$ of $H^{n^{\prime}}, n^{\prime}=n+1$ with parameters

$$
\left(\begin{array}{cccc}
0 & n^{\prime} & 0 & 0 \\
2 & 0 & n^{\prime}-2 & 0 \\
0 & n^{\prime}-2 & 0 & 2 \\
0 & 0 & n^{\prime} & 0
\end{array}\right)
$$

as follows:

$$
\begin{aligned}
G_{1} & =\left\{\bar{x} \alpha \mid \bar{x}=x_{1} x_{2} \ldots x_{n} \in C_{12}, \alpha=x_{1}+\ldots+x_{n} \bmod 2\right\} \\
G_{4} & =\left\{\bar{x} \beta \mid \bar{x}=x_{1} x_{2} \ldots x_{n} \in C_{4}, \beta=x_{1}+\ldots+x_{n}+1 \bmod 2\right\} \\
G_{2} & =\left\{\bar{y} \in V\left(H^{n^{\prime}}\right) \mid d\left(\bar{y}, C_{1}\right)=1\right\} \\
G_{3} & =\left\{\bar{y} \in V\left(H^{n^{\prime}}\right) \mid d\left(\bar{y}, C_{4}\right)=1\right\} .
\end{aligned}
$$

This partition can be viewed as an "extended" version of the partition $\left\{C_{12}, C_{3}, C_{4}\right\}$; the spittability of $C_{12}$ or $C_{4}$ is equivalent to the spittability of $G_{1}$ or $G_{4}$ respectively. But the distance 1 between vertices of, say, $C_{4}$ corresponds to the distance 2 between the corresponding vertices of $G_{4}$; and the graph of distances 1 and 2 of $C_{4}$ corresponds to the graph of distances 2 of $G_{4}$, which emphasize the "equal status" of the all edges of the graph. Of cause if $G_{1}$ and/or $G_{4}$ are splittable, then splitting gives an equitable partition of $H^{n^{\prime}}$ into $6 / 5$ parts with corresponding parameters. If both $G_{1}$ and $G_{4}$ are splittable (say, into $G_{1}^{\prime}, G_{1}^{\prime \prime}$ and $G_{4}^{\prime}, G_{4}^{\prime \prime}$ respectively), then the equitable partition $\left\{G_{1}, G_{2}, G_{3}, G_{4}\right\}$ (defined in some other terms) is also known as a code-generating factorization of $H^{n^{\prime}}$ [14. Indeed, the code $G_{1}^{\prime} 0 \cup G_{1}^{\prime \prime} 1 \cup G_{4}^{\prime} 0 \cup G_{4}^{\prime \prime} 1$ is 1-perfect.

\section{$3 \quad$ Embedding in twofold 1-perfect codes}

Theorem 2. Let $C_{1}$ be a $\left(n=2^{m}-3,2^{2^{m}-m-3}, 3\right)$ code. Then the set $C_{1} 00=$ $\left\{\bar{x} 00 \mid \bar{x} \in C_{1}\right\}$ is a subset of a unique twofold 1-perfect code $B$ with the following properties:

a) the multiplicity of any codeword of $C_{1} 00$ is 2 ;

b) any codeword $\bar{x}$ with the last two symbols 01 or 10 satisfies $\bar{x}+0 \ldots 011 \in C$. 
Proof: Existence. Let $B=2 * C_{1} 00 \cup 2 * C_{2} 11 \cup C_{4} 01 \cup C_{4} 10$. Obviously, $B$ satisfies a), b), and $C_{1} 00 \subset B$. The fact that $B$ is a twofold 1-perfect code is straightforward from Theorem 1 we leave the details as an exercise.

Uniqueness. Assume $B$ is a twofold 1-perfect code satisfying a), b), and $C_{1} 00 \subset B$. Define

$$
\begin{aligned}
& C_{2}=\{\bar{x} \mid \bar{x} 11 \in B\}, \\
& C_{4}=\{\bar{x} \mid \bar{x} 01 \in B\}, \\
& C_{3}=V\left(H^{n}\right) \backslash\left(C_{1} \cup C_{2} \cup C_{4}\right)
\end{aligned}
$$

From the antipodality of $B$, we have $C_{2}=C_{1}$. As follows from the definition of twofold 1-perfect codes, any codeword of multiplicity 2 cannot be at distance 1 or 2 from any other codeword. Consequently, 1) the distance between $C_{1}$ and $C_{4}$, as well as between $C_{2}$ and $C_{4}$, cannot be less than $2 ; 2$ ) the multiplicity of the words of form $\bar{x} 01$ in $B$ is less than 2 .

Now we see that, by numerical reasons, $C_{4}$ consists of the all vertices at the distance more than 1 from $C_{1}$. Thus, $C_{2}, C_{3}$, and $C_{4}$ satisfy (1)-(3), and $B$ is unique. $\triangle$

By similar arguments, the following is also true:

Theorem 3. Let $C_{1}$ be a $\left(n=2^{m}-3,2^{2^{m}-m-3}, 3\right)$ code. Then the set $C_{1} 00$ is a subset of a unique twofold 1-perfect code $D$ whose all codewords $\bar{x}$ satisfy $\bar{x}+0 \ldots 011 \in C$.

\section{Embedding in 1-perfect codes}

Theorem 4. Let $C_{1}$ be a $\left(n=2^{m}-3,2^{2^{m}-m-3}, 3\right)$ code. The following four statements are mutually equivalent:

a) the set $C_{1} 00$ is a subset of a 1-perfect code $C$;

b) the set $C_{4}$ defined in (3) is splittable;

c) the twofold 1-perfect $B$ from Theorem 2 is splittable;

d) the twofold 1-perfect $D$ from Theorem 3 is splittable.

Proof: Clearly, each of c) and d) implies a).

Since $B$, as well as $D$, includes $C_{4} 01$, each of c) and d) implies b).

Conversely, assume b) holds and $C_{4}=C^{\prime} \cup C^{\prime \prime}$ where $C^{\prime}$ and $C^{\prime \prime}$ are distance3 codes. Then

$$
\begin{aligned}
& B=\left(C_{1} 00 \cup C_{4} 11 \cup C^{\prime} 01 \cup C^{\prime \prime} 10\right) \cup\left(C_{1} 00 \cup C_{4} 11 \cup C^{\prime} 10 \cup C^{\prime \prime} 01\right), \\
& D=\left(C_{1} 00 \cup C_{4} 11 \cup C^{\prime} 01 \cup C^{\prime \prime} 10\right) \cup\left(C_{4} 00 \cup C_{1} 11 \cup C^{\prime} 10 \cup C^{\prime \prime} 01\right),
\end{aligned}
$$

and c), d) hold.

Assume a) is true. Define $C^{\prime}=\{\bar{x} \mid \bar{x} 01 \in C\}$ and $C^{\prime \prime}=\{\bar{x} \mid \bar{x} 10 \in C\}$. Because of the code distance 3 of $C$, we see that $C^{\prime}$ and $C^{\prime \prime}$ are disjoint and at the distance at least 2 from $C_{1}$. So, since $\left|C^{\prime}\right|+\left|C^{\prime \prime}\right|=\left|C_{4}\right|$, we get $C_{4}=C^{\prime} \cup C^{\prime \prime}$, and b) holds. $\triangle$ 
Remark 3. The splittability of any of the sets $C_{4}, B, D$ is equivalent to the biparticity of its graph of distances 1 and 2 (two codewords $\bar{x}$ and $\bar{y}$ are adjacent if and only if $d(\bar{x}, \bar{y}) \in\{1,2\})$. In this graph for $D$, the vertices of types $\bar{x} 00$ and $\bar{x} 11$ are not connected with the vertices of types $\bar{x} 01$ and $\bar{x} 10$, and the subgraph generated by the former vertices is bipartite, while the biparticity of the remaining subgraph is questionable. In $B$, the codewords of types $\bar{x} 00$ and $\bar{x} 11$ have the multiplicity 2 , and they are isolated in the graph of distances 1 and 2 .

Remark 4. If $\nu$ is the number of connected components in the graph of distances 1 and 2 of $C_{4}$, then the number of different 1-perfect codes including $C_{1} 00$ is $2^{\nu}$. As follows from the tight lower bound on the size of the difference between two 1-perfect codes [13, 3], the cardinality of a connected component is not less than $2^{\frac{n-1}{2}}$, and so $\nu \geq \frac{2^{\frac{n-3}{2}}}{n+1}$. If $C_{1}$ is linear, then $\nu$ achieves this bound.

\section{$5 \quad$ Unsplittable twofold STS}

If we consider a 1-perfect code containing $\overline{0}$, then all the weight-3 codewords compose a design known as a Steiner triple system, or STS. The characteristic property of an $S T S$ is that every weight-2 word is at distance 1 from exactly one word of the STS. (Strictly speaking, an STS is defined as a pair $(V, B)$, where $V$ is some set and $B$ is a collection of 3-subsets of $V$, named blocks, such that every 2-subset of $V$ is included in exactly one block.)

If we consider a twofold 1-perfect code $C$ such that the multiplicity of $\overline{0}$ is 2 , then all the weight- 3 codewords compose a design, which can be called a twofold STS. If $C$ comes from Theorem 2, then the corresponding STS satisfies

a) any codeword of type $\bar{x} 00$ or $\bar{x} 11$ has the multiplicity 2 ;

b) for any $\bar{x}$ of the corresponding length, $\bar{x} 01$ and $\bar{x} 10$ are codewords or not simultaneously.

For the length 15, there exists a twofold STS meeting a) and b) that cannot be split into two STS. This fact has not direct connection with the problem considered in this paper: on one hand, it is not proved that there exists a twofold 1-perfect code that include this STS (e.g., for the length 15, there exist STSs that are not embeddable in a 1-perfect code [10]); on the other hand, the splittability of the all twofold STS included in a twofold 1-perfect code would not mean the splittability of the twofold 1-perfect itself. Nevertheless, the existence of such an object seems to be interesting. The following is the list of the words of the mentioned example (the unsplittability follows from the existence of a 5 -cycle in the distance-2 graph):

$\begin{array}{ll}000000000000111 \times 2, & \\ 000000011000001, & 000000011000010, \\ 000000001100001, & 000000001100010, \\ 000000000110001, & 000000000110010, \\ 00000000011001, & 00000000011010, \\ 000000010001001, & 000000010001010, \\ 11000000000001, & 1100000000010, \\ 01100000000001, & 01100000000010, \\ 001100000000001, & 001100000000010,\end{array}$




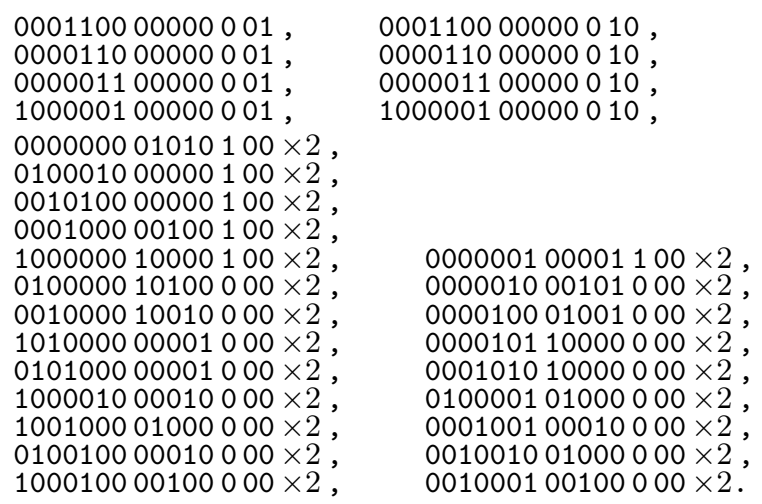

$000000100001100 \times 2$, $000001000101000 \times 2$, $000010001001000 \times 2$, $000010110000000 \times 2$, $000101010000000 \times 2$, $010000101000000 \times 2$, $000100100010000 \times 2$, $001001001000000 \times 2$, $001000100100000 \times 2$.

\section{MDS codes and double-MDS-codes}

Let $Q^{m}=\left(V\left(Q^{m}\right), E\left(Q^{m}\right)\right)$ denotes the graph whose vertex set is the set $\{0,1,2,3\}^{m}$ of quaternary $n$-words, two words being adjacent if and only if they differ in exactly one position. By a 4-clique we mean a set of four words of $V\left(Q^{m}\right)$ differing in exactly one position.

A subset $M$ of $V\left(Q^{m}\right)$ is called an MDS code (with distance 2) if every 4-clique contains exactly one word of $M$. Equivalently, $M$ is a distance 2 code of cardinality $4^{m-1}$. Equivalently, $\left\{M, V\left(Q^{m}\right) \backslash M\right\}$ is an equitable partition of $Q^{m}$ with parameter matrix $((0,3 m)(m, 2 m))$.

We call a subset $M$ of $V\left(Q^{m}\right)$ a double-MDS-code if every 4-clique contains exactly two word of $M$. Equivalently, $\left\{M, V\left(Q^{m}\right) \backslash M\right\}$ is an equitable partition of $Q^{m}$ with parameter matrix $((m, 2 m)(2 m, m))$.

A double-MDS-code is splittable if it is the union of two (disjoint) MDS codes.

Denote $P_{0}=\{0000,1111\}, P_{1}=\{0011,1100\}, P_{2}=\{0101,1010\}, P_{3}=$ $\{0110,1001\} \subset V\left(H^{4}\right)$ and $P_{0}^{\prime}=\{000,111\}, P_{1}^{\prime}=\{011,100\}, P_{2}^{\prime}=\{101,010\}$, $P_{3}^{\prime}=\{110,001\} \subset V\left(H^{3}\right)$. Let $C \in V\left(H^{m-1}\right)$ be a 1-perfect binary code; denote $C^{*}=\left\{000 c_{1} 000 c_{2} \ldots 000 c_{m-1} 000 \mid c_{1} c_{2} \ldots c_{m-1} \in C\right\}$. For any subset $M$ of $V\left(Q^{m}\right)$ we define the code $S(M) \subset V\left(H^{4 m-1}\right)$ as follows:

$$
S(M)=\bigcup_{\mu_{1} \ldots \mu_{m} \in M} P_{\mu_{1}} P_{\mu_{2}} \ldots P_{\mu_{m-1}} P_{\mu_{m}}^{\prime}+C^{*}
$$

Here, for two sets of words $P^{1} \subset V\left(H^{r}\right)$ and $P^{2} \subset V\left(H^{l}\right), P^{1} P^{2}=\left\{x_{1} \ldots x_{r} y_{1} \ldots y_{l} \mid\right.$ $\left.x_{1} \ldots x_{r} \in P^{1}, y_{1} \ldots y_{l} \in P^{2}\right\}$; and if $r=l$, then $P^{1}+P^{2}=\left\{z_{1} \ldots z_{r} \mid z_{i}=\right.$ $\left.x_{i}+y_{i} \bmod 2, x_{1} \ldots x_{r} \in P^{1}, y_{1} \ldots y_{r} \in P^{2}\right\}$.

Proposition 2. 1) If the code distance of $M$ is not less than 2, then the code distance of $S(M)$ is at least 3; if $M$ is an MDS code, then $S(M)$ is a 1-perfect code. 2) If $M$ is a splittable (unsplittable) double-MDS-code, then $S(M)$ is a splittable (unsplittable) twofold 1-perfect code.

Proof (a sketch): P. 1) is proved in [11, in more general form. 
Similarly, if $M$ is a double-MDS-code, then $S(M)$ is a twofold 1-perfect code (it is straightforward to check the definition). If $M=M^{\prime} \cup M^{\prime \prime}$ for some MDS codes $M^{\prime}$ and $M^{\prime \prime}$, then $S(M)=S\left(M^{\prime}\right) \cup S\left(M^{\prime \prime}\right)$, where $S\left(M^{\prime}\right)$ and $S\left(M^{\prime \prime}\right)$ are 1-perfect codes. Otherwise, the distance-1 graph of $M$ has an odd cycle, and it is easy to find a corresponding cycle of the same length in the graph of distances 1 and 2 of $S(M)$, which implies that $S(M)$ is unsplittable. $\triangle$

Theorem 5. Let $m=2^{k-2}$. Assume there exists an unsplittable double-MDScode $M_{1} \subset V\left(Q^{m-1}\right)$ such that the double-MDS-code $M_{0}=V\left(Q^{m-1}\right) \backslash M_{1}$ is splittable. Then there exist a $\left(n=2^{k}-3,2^{2^{k}-k-3}, 3\right)$ code $C_{1}$ such that $C_{1} 00$ is not a subset of a 1-perfect code.

Proof: Let $M_{0}=M^{\prime} \cup M^{\prime \prime}$ where $M^{\prime}$ and $M^{\prime \prime}$ are disjoint MDS codes.

Denote $M=M_{0} 0 \cup M_{0} 1 \cup M_{1} 2 \cup M_{1} 3 \subset V\left(Q^{m}\right)$. By the definition, $M$ is a double-MDS-code. Since $M_{1}$ is unsplittable, $M$ is unsplittable too. Then, by Proposition 2, the set

$$
D=S(M)
$$

is an unsplittable twofold 1-perfect code.

Now, consider the set

$$
C=S\left(M^{\prime} 0 \cup M^{\prime} 1\right) .
$$

Since the code distance of $M^{\prime} 0 \cup M^{\prime} 1$ is 2, the code distance of $C$ is at least 3, by Proposition 2. Half of the codewords of $C$ have 00 in the last two positions (the others, 11); let $C_{1} 00$ denote the corresponding subcode.

We have: $\left|C_{1}\right|=\frac{1}{8}|C|=2^{2^{k}-k-3}$; the code distance of $C_{1}$ is $3 ; C_{1} 00 \subset D$ where $D$ is an unsplittable twofold 1-perfect code whose all codewords $\bar{x}$ satisfy $\bar{x}+0 \ldots 011 \in C$. By Theorems 3 and 4 , the proof is over. $\triangle$

Conjecture (V. Potapov). Any double-MDS-code $M$ in $Q^{m}$ is splittable if and only if its complement $V\left(Q^{m}\right) \backslash M$ is splittable.

This is equivalent to the following statement: Any $4 \times 4 \times \ldots 4 \times 2$ latin hypercuboid is completable to $a 4 \times 4 \times \ldots 4 \times 4$ latin hypercube. A $q \times q \times$ $\ldots q \times p$ latin hypercuboid of order $q$ (if $p=q$, latin hypercube) is a function $f:\{0, \ldots, q-1\}^{m-1} \times\{0, \ldots, p-1\} \rightarrow\{0, \ldots, q-1\}$ such that $f(\bar{x}) \neq f(\bar{y})$ for any $\bar{x}$ and $\bar{y}$ differing in exactly one position. Examples of non-completable latin cuboids are constructed in [5, 9]

Another equivalent formulation: Let $K_{4}^{m}$ be the direct product of $m$ copies of the complete graph on 4 vertices. If $V\left(K_{4}^{m}\right)$ is partitioned into two subsets that generate subgraphs of degree $m$, then these subgraphs are bipartite or not bipartite simultaneously.

It seems perspective to use the characterization of the distance-2 MDS codes over the quaternary alphabet (latin hypercubes of order 4) 7 to prove this conjecture. Nevertheless, the analysis of all subcases needs some work, which is not completed at this moment. In any case, it is interesting to find an independent proof. 


\section{References}

[1] M. R. Best and A. E. Brouwer. The triply shortened binary Hamming code is optimal. Discrete Math., 17(3):235-245, 1977 . DOI: 10.1016/0012-365X(77)90158-3.

[2] T. Blackmore. Every binary $\left(2^{m}-2,2^{2^{m}-2}-m, 3\right)$ code can be lengthened to form a perfect code of length $2^{m}-1$. IEEE Trans. Inf. Theory, 45(2):698700, 1999. DOI: 10.1109/18.749014.

[3] T. Etzion and A. Vardy. Perfect binary codes: Constructions, properties and enumeration. IEEE Trans. Inf. Theory, 40(3):754-763, 1994. DOI: $10.1109 / 18.335887$.

[4] T. Etzion and A. Vardy. On perfect codes and tilings: Problems and solutions. SIAM J. Discrete Math., 11(2):205-223, $1998 . \quad$ DOI: 10.1137/S0895480196309171.

[5] M. Kochol. Relatively narrow Latin parallelepipeds that cannot be extended to a Latin cube. Ars Combin., 40:247-260, 1995.

[6] D. S. Krotov and V. N. Potapov. On multifold MDS and perfect codes that are not splittable into onefold codes. Probl. Inf. Transm. 40(1):5-12, 2004. DOI: 10.1023/B:PRIT.0000024875.79605.fc translated from Probl. Peredachi Inf. 40(1) (2004), 6-14.

[7] D. S. Krotov and V. N. Potapov. n-Ary quasigroups of order 4. SIAM J. Discrete Math., 23(2):561-570, 2009. DOI: 10.1137/070697331. ArXiv:math/0701519

[8] S. P. Lloyd. Binary block coding. Bell Syst. Tech. J., 36(2):517-535, 1957.

[9] B. D. McKay and I. M. Wanless. A census of small Latin hypercubes. SIAM J. Discrete Math., 22(2):719-736, 2008. DOI: 10.1137/070693874

[10] P. R. J. Östergård and O. Pottonen. There exist Steiner triple systems of order 15 that do not occur in a perfect binary one-error-correcting code. J. Comb. Des., 15(6):465-468, 2007. DOI: 10.1002/jcd.20122.

[11] K. T. Phelps. A general product construction for error correcting codes. SIAM J. Algebraic Discrete Methods, 5(2):224-228, 1984.

[12] H. S. Shapiro and D. L. Slotnick. On the mathematical theory of error correcting codes. IBM J. Res. Develop., 3(1):25-34, 1959.

[13] F. I. Solov'eva. Exact bounds on the connectivity of code-generating disjunctive normal forms. Preprint 10, Inst. Math. of the Siberian Branch of Acad. of Sciences USSR, 1990. 
[14] Yu. L. Vasil'ev and F. I. Solov'eva. Codegenerating factorization on $n$ dimensional unite cube and perfect binary codes. Probl. Inf. Transm. 33(1):64-74, 1997. Translated from Probl. Peredachi Inf. 33(1) (1997), 64-74. 